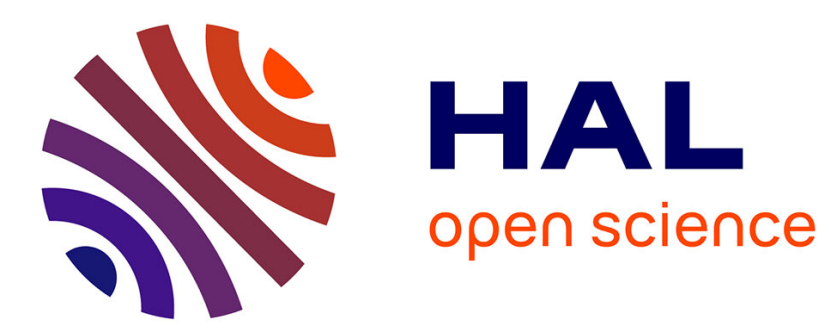

\title{
Further Steps Towards the Development of a Hardware Simulator for MIMO Radio Channels
}

\author{
Sylvie Picol, Gheorghe Zaharia, Ghaïs El Zein
}

\section{To cite this version:}

Sylvie Picol, Gheorghe Zaharia, Ghaïs El Zein. Further Steps Towards the Development of a Hardware Simulator for MIMO Radio Channels. IEEE 16th International Symposium on Personal, Indoor and Mobile Radio Communications, 2005. PIMRC 2005., Sep 2005, France. p. 557 - 561. hal-00175823

\section{HAL Id: hal-00175823 \\ https://hal.science/hal-00175823}

Submitted on 1 Oct 2007

HAL is a multi-disciplinary open access archive for the deposit and dissemination of scientific research documents, whether they are published or not. The documents may come from teaching and research institutions in France or abroad, or from public or private research centers.
L'archive ouverte pluridisciplinaire HAL, est destinée au dépôt et à la diffusion de documents scientifiques de niveau recherche, publiés ou non, émanant des établissements d'enseignement et de recherche français ou étrangers, des laboratoires publics ou privés. 


\title{
Further Steps Towards the Development of a Hardware Simulator for MIMO Radio Channels
}

\author{
Sylvie Picol, Gheorghe Zaharia, Member, IEEE, Ghaïs El Zein
}

\begin{abstract}
The aim of the regional project SIMPAA2, which continues the research activities of the former national research SIMPAA project, is the realization of a hardware simulator of MIMO propagation channels for UMTS and WLAN applications. The simulator must reproduce the behavior of the radio propagation channel, thus making it possible to test "on table" the mobile radio equipments. The advantages are: low cost, short test duration, possibility to ensure the same test conditions in order to compare the performance of various equipments.
\end{abstract}

Index Terms-hardware simulator, MIMO propagation channel, UMTS, WLAN.

\section{INTRODUCTION}

$\mathrm{D}$ URING last years, the development of wireless technologies offers many prospects and new applications. This trend will probably be accentuated in the future, the systems still having to improve their data rate and mobility. UMTS (Universal Mobile Telecommunications System) and WLAN (Wireless Local Area Networks) networks constitute the mobile and wireless telecommunications systems of third generation able to offer to general public the high-rate multimedia services. The capacity improvement of wireless networks has drawn considerable attention to MIMO (Multiple-Input Multiple-Output) communication techniques. MIMO systems make use of antenna arrays at both the transmit and the receive sides of a radio link to drastically improve the capacity over more traditional systems.

However, the transmitted electromagnetic waves interact with the propagation environment (indoor/outdoor). It is thus necessary to take into account the main propagation parameters during the design of the future communication systems. The optimal choice of the modulation, coding, etc. for these communication systems is based on a reliable model of the radio propagation channel. Moreover, after the realization of a communication system, its experimental performance can be evaluated by using a hardware radio channel simulator. A hardware simulator can also be used to compare the performance of various radio communication

Manuscript received March 1, 2005. This work is supported by the "Conseil Régional de Bretagne" under the regional research project SIMPAA2.

S. Picol, G. Zaharia and G. El Zein are with the Institut d'Electronique et de Télécommunication de Rennes, UMR CNRS 6164, INSA de Rennes, France (phone: (+33) 2-23-23-87-14; fax: (+33) 2-23-23-84-39; e-mail: sylvie.picol@ens.insa-rennes.fr). systems in a time-variant propagation channel in the same test conditions.

This article presents the design of a hardware simulator for indoor/outdoor MIMO radio channels. Section 2 describes the classification of the environments and the main propagation phenomena. Section 3 deals with the design of the channel simulator. It is divided into three parts : Channel model, RF and digital blocks describing their respective architecture and several parameters useful to design the hardware simulator. Lastly, Section 4 shows the software simulation of a SISO (Single-Input Single-Output) propagation channel. This simulation allows the optimal choice of several parameters: number of bits per sample, data format (floating-point, fixedpoint), truncation introducing an error, etc. The trade-off between results accuracy and complexity represents an important choice during the design of the hardware simulator. This software simulation is an essential step before the hardware implementation.

\section{Propagation EnVironment}

The development of a radio communication system requires the knowledge of the main characteristics of the propagation channel.

Within the context of wireless communications in UMTS networks, the propagation environments can be classified in several cells categories [1], differentiated by their size, traffic, number and dimensions of the obstacles liable to disturb the electromagnetic wave propagation (Fig. 1).

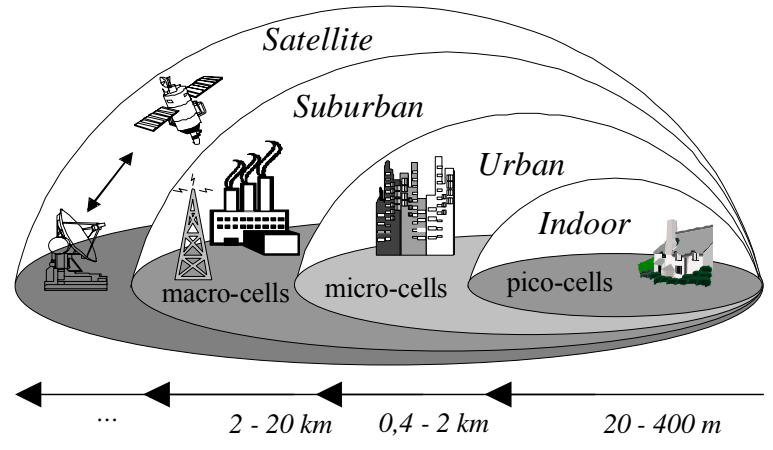

Fig. 1. Radio propagation environments.

Three main categories can be distinguished: pico-cells which concern communications inside a building, micro-cells which refer to communications on a district scale (or a small city), and finally macro-cells which include cities and their 
neighborhoods. The specific characteristics of these environments determine the operation of the communication systems. The larger the cell size is, the more the delays are significant and the received power is reduced. Consequently, it is more difficult to reach good performances especially when high data rate must be obtained.

In these environments, the main physical phenomena affecting the transmitted signal are the following:

- reflection: it appears when the wave impinges upon an object whose size is larger than the wavelength and the irregularities of surface smaller than this same wavelength. Then, the incident wave is partly reflected and the other part is refracted (Fig. 2.A). The refraction describes the wave transmitted through the obstacle. If the obstacle is perfectly conductive, there is no transmitted wave, all energy is reflected,

- diffusion: when reflection occurs on an object whose surface roughness has dimensions comparable with the wavelength, the reflection becomes diffuse, i.e. incident energy is radiated in all the directions (Fig. 2.B),

- diffraction: it appears when the electromagnetic wave is disturbed by the presence of an obstacle presenting irregular forms (edge, points, cones...) between a transmitter and a receiver. Nevertheless, this phenomenon can present positive aspects because it allows radio signals to propagate around the curved surface of the earth, beyond the horizon, and to propagate behind the obstructions (Fig. 2.C).

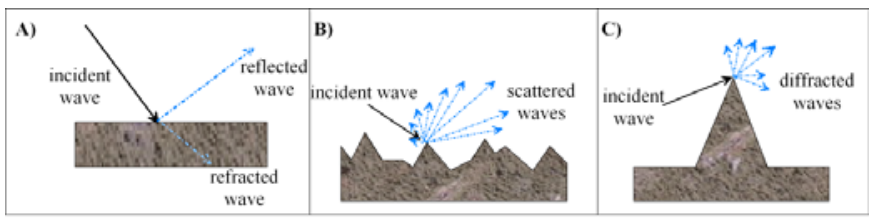

Fig. 2. Propagation phenomena.

Due to the radio propagation phenomena, the received signal is a combination of several paths of different amplitudes, delays, phases and angles of arrivals: it is the multipath propagation. These paths are mainly due to the wave reflections on the obstacles present within the propagation environment: buildings, trees, mountains. This phenomenon is illustrated very schematically on Fig. 3.

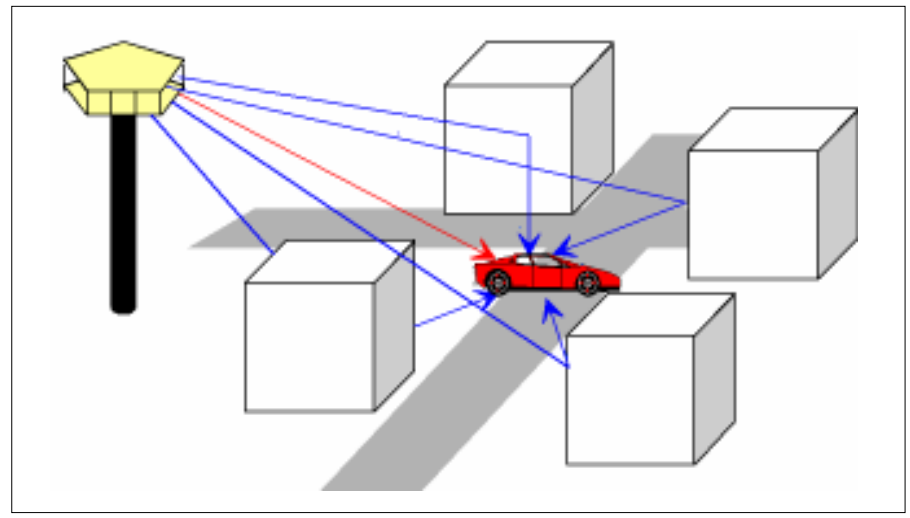

Fig. 3. Multipath propagation (a direct path and several reflections).
It is one of the phenomena that contribute to deteriorate the received signal. In addition, the transmitter and/or the receiver, as well as the obstacles (vehicles, pedestrians, etc.), can be mobile. Thus, the various paths will evolve in time, some of them being able to appear or disappear: the channel is time-variant.

Moreover, the multipath propagation involves fading [2] and spread of the received signal in various fields:

- the RMS delay spread is due to the differences between the delays of different paths. It is related to the coherence bandwidth and gives information on the frequency selectivity of the radio propagation channel,

- the spread of the AOA (angle of arrival) is due to the directions of arrival of different paths,

- the Doppler spread which depends on the relative mobility of the transmitter-receiver-scatterers and determines the coherence time.

\section{Hardware Simulator: Principle, Channel MODELING AND OPERATION}

The simulator must reproduce the behavior of a MIMO propagation channel for a specific environment, indoor or outdoor. This makes it possible to check the correct operation of the new radio communication systems and to compare their performances under the same test conditions.

The simulator must operate with RF signals (2 GHz for UMTS and $5 \mathrm{GHz}$ for WLAN). In order to make adjacent channels interference tests for UMTS systems, it is useful to consider three successive channels, thus to have a $3 \times 5 \mathrm{MHz}$ bandwidth. The frequency bandwidths $\mathrm{B}$ are: $15 \mathrm{MHz}$ for UMTS and $20 \mathrm{MHz}$ for WLAN.

Moreover, UMTS uses two operating modes: the TDD mode (Time Division Duplex) having the same frequency band for both uplink and downlink and the FDD mode (Frequency Division Duplex) having different frequency bands. In addition, depending on the strength of the transmitted signals, the simulator must be able to accept input signals with wide power range, between - 50 and $33 \mathrm{dBm}$, which implies a power control for the simulator inputs.

The number of radio frequency blocks is determined by the number of the transmitting and receiving antennas. For a transmitter with $\mathrm{N}_{T}$ antennas and a receiver using $N_{R}$ antennas, the MIMO simulator must have $\mathrm{N}_{T} \mathrm{~N}_{R}$ full-duplex SISO channels, thus $2 \mathrm{~N}_{\mathrm{T}} \mathrm{N}_{\mathrm{R}}$ one-way SISO channels. The choice of 16 full-duplex SISO channels seems to be a suitable trade-off between utility and complexity, assuming $\mathrm{N}_{\mathrm{T}}, \mathrm{N}_{\mathrm{R}} \leq 8$ and $\mathrm{N}_{\mathrm{T}} \mathrm{N}_{\mathrm{R}} \leq 16$.

The design and realization of the RF block for UMTS systems were completed in the previous SIMPAA (SImulateur Matériel de Propagation pour Antennes Adaptatives) project [3]. The RF block will need some modifications required by WLAN specifications. The RF blocks allow, for the downlink, to transpose the received signal to the intermediate frequency (IF), then to a bandwidth B low-pass signal, and conversely for the uplink.

The objectives of the regional SIMPAA2 project mainly concern the channel model block and the digital block of the 
MIMO simulator, as shown in Fig. 4 by the gray blocks.

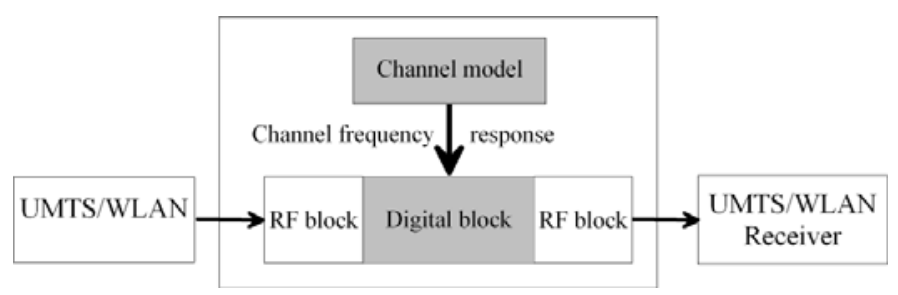

Fig. 4. Block diagram of a one-way SISO channel.

\section{A. Channel Model Block}

A MIMO channel is composed of several randomly timevariant correlated SISO channels. Fig. 5 illustrates a MIMO channel with $\mathrm{N}_{T}=2$ transmit antennas and $\mathrm{N}_{\mathrm{R}}=2$ receive antennas.

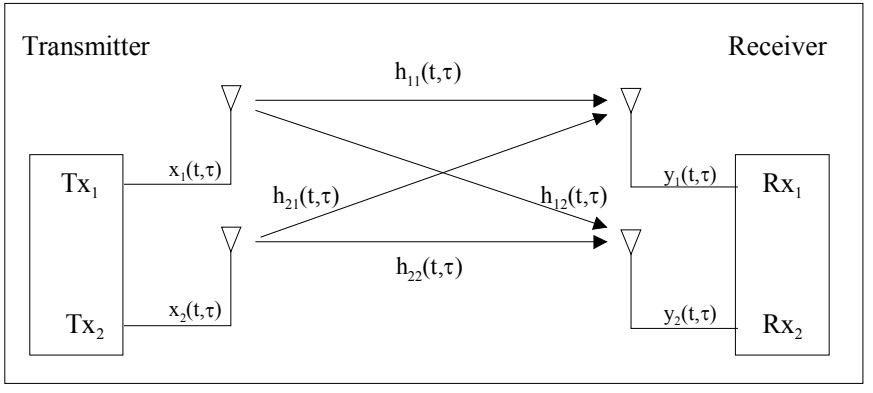

Fig. 5. MIMO channel ( $2 \times 2$ SISO channels $)$.

For this MIMO channel, the signal $\mathrm{y}_{\mathrm{j}}(\mathrm{t}, \tau)$ received by the $\mathrm{j}^{\text {th }}$ receive antenna can by easily obtained:

$$
\mathrm{y}_{\mathrm{j}}(\mathrm{t}, \tau)=\mathrm{x}_{1}(\mathrm{t}, \tau) * \mathrm{~h}_{1 \mathrm{j}}(\mathrm{t}, \tau)+\mathrm{x}_{2}(\mathrm{t}, \tau) * \mathrm{~h}_{2 \mathrm{j}}(\mathrm{t}, \tau), \mathrm{j}=1,2
$$

For a hardware implementation, it is easier to use the Fourier transform to replace the convolution by an algebraic product:

$$
Y_{j}(t, f)=X_{1}(t, f) \cdot H_{1 j}(t, f)+X_{2}(t, f) \cdot H_{2 j}(t, f), j=1,2
$$

Each randomly time-variant SISO channel is described by one of the Bello's characteristic functions [4], as shown in Fig. 6.

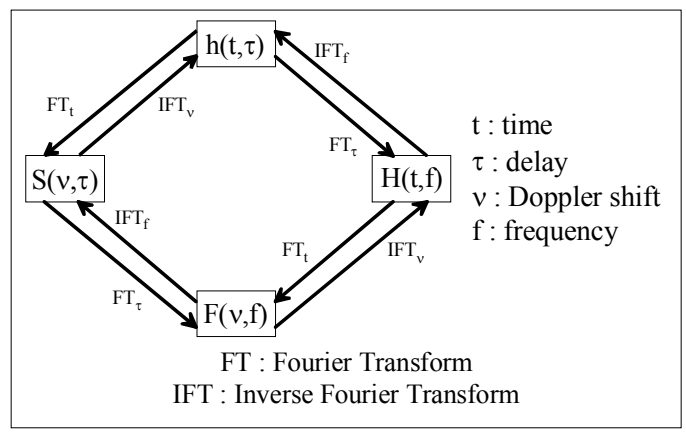

Fig. 6. Bello's characteristic functions.

The knowledge of one characteristic function makes it possible to compute the other characteristic functions by using the direct or inverse Fourier Transform.
There are several classes of channel models [5]: the parametric and nonparametric models and, in each one of these classes, the deterministic and stochastic models. The stochastic parametric models for different typical environments describe the parameters of the channel model by statistical laws, obtained by the analysis of measured data.

There are two types of channel sounders: the time domain sounders which measure the channel impulse response and the frequency domain sounders which give the channel frequency response.

The channel models used by the simulator will be obtained from measurements by using a time domain MIMO channel sounder designed and realized at the IETR [6], as shown in Fig. 7.

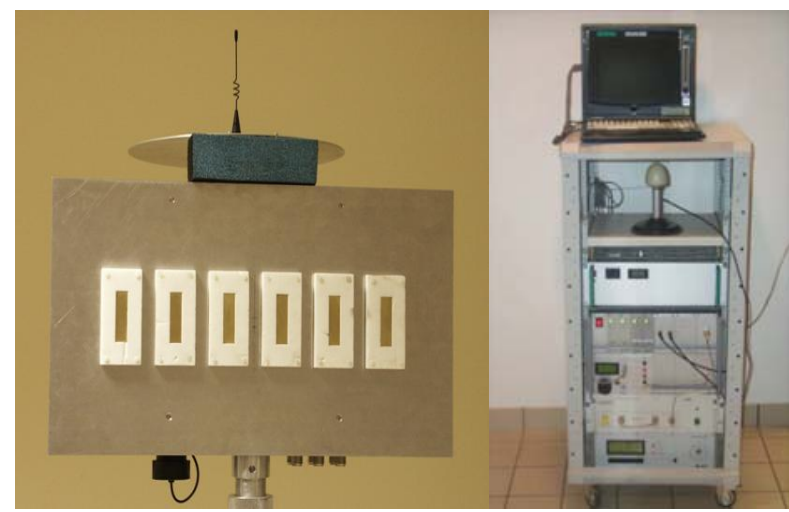

Fig. 7. Antennas array and the transmitter of the MIMO channel sounder.

This MIMO channel sounder is based on the correlation technique [1]. For each SISO channel, the sounder gives the measured complex envelope of its impulse response. During the measurement of all SISO channels, the MIMO channel must be practically invariant. This limits the mobile speed and therefore the maximal value of the Doppler shift.

The simplest way to use these measurement results is to compute, for each location of the mobile transmitter, the frequency responses $\mathrm{H}_{\mathrm{i}, \mathrm{j}}(\mathrm{t}, \mathrm{f})$ as Fourier transforms of the impulse responses $h_{i, j}(t, \tau)$ of the MIMO propagation channel. These frequency responses can be used to supply the "Channel model" block of each SISO channel, as shown in Fig. 4. Periodically, the frequency responses must be actualized, in order to simulate the channel time-evolution. The "refreshing" period of the frequency responses is determined by the channel coherence time, thus by the mobile speed and the propagation environment. As the number of the recorded files is finite, it will be necessary to periodically replay the whole set of frequency responses.

A more elaborate way to use the measurement results is to determine a statistical MIMO channel for each type of propagation environment (indoor, urban, rural, mountainous, etc.) and to generate $h_{i, j}(t, \tau)$ profiles within the digital block. In this case, local FFT (Fast Fourier Transform) modules are required to compute the frequency responses $\mathrm{H}_{\mathrm{i}, \mathrm{j}}(\mathrm{t}, \mathrm{f})$ to be used by (2). Finally, for each receive antenna, an IFT will be used to obtain the time response $\mathrm{y}_{\mathrm{j}}(\mathrm{t}, \tau)$. 


\section{B. RF Block}

Fig. 8 shows the realization of the RF reception (high part) and transmission (low part) units, as well as the connection unit (left part) which allows the link between antenna and the $\mathrm{RF}$ reception and transmission units. This RF block was realized in printed technology during the previous SIMPAA project.

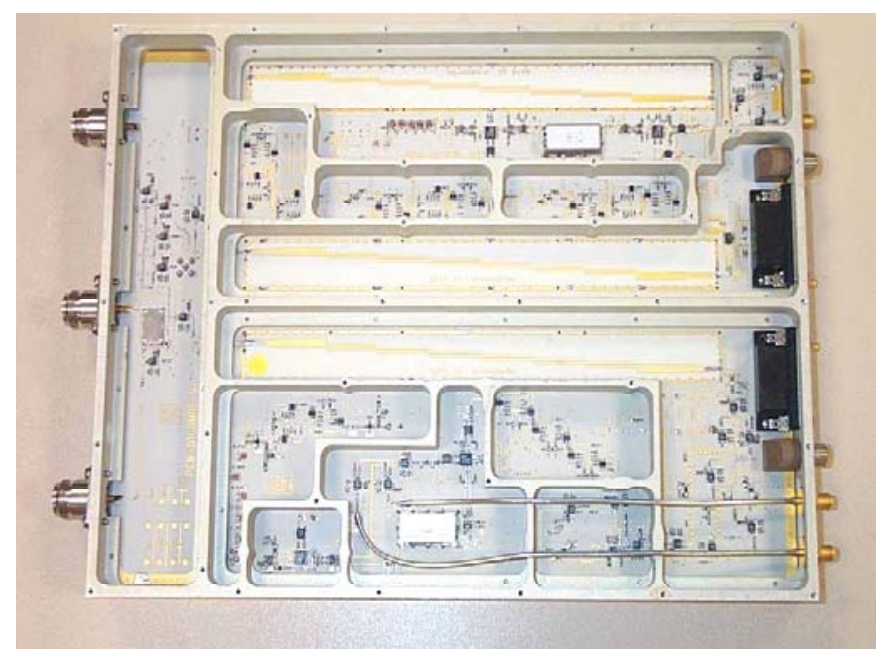

Fig. 8. UMTS reception and transmission of a SISO RF block

As shown in Fig. 9, for RF receiver and transmitter, the blocks "Attenuator" and "Amplifier" are controlled respectively via "Control bus" and "Gain control bus" in order to adjust the reception and transmission power by the automatic gain control (AGC).

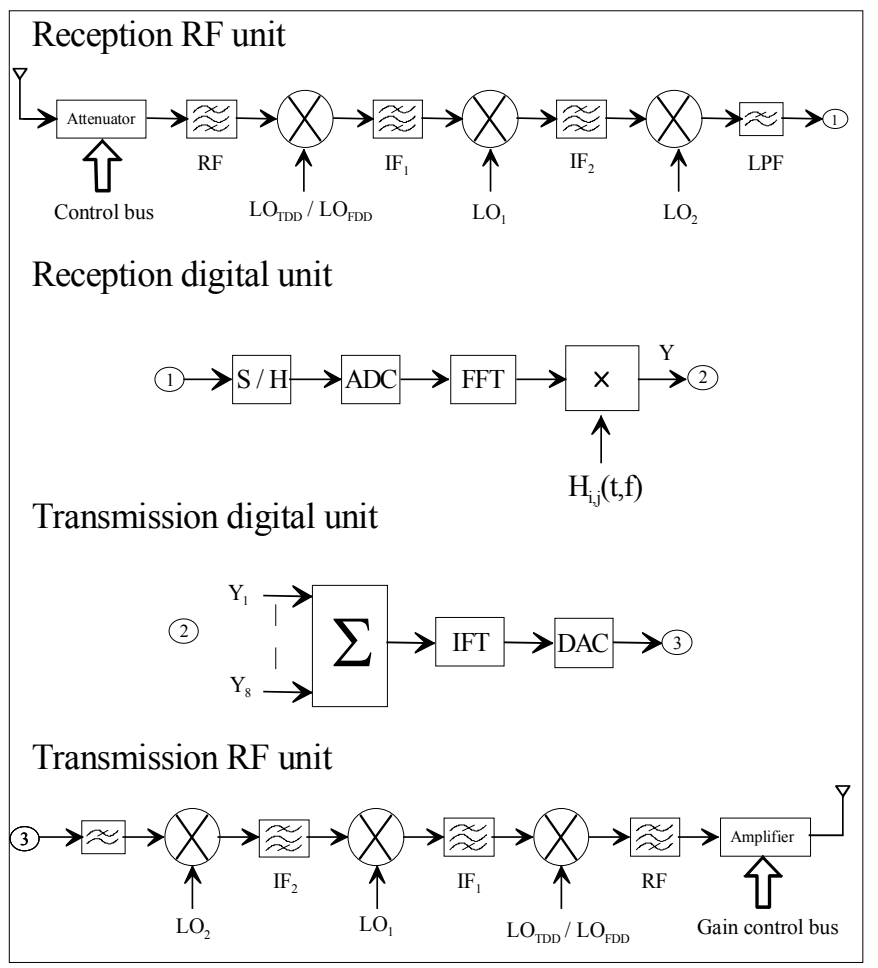

Fig. 9. RF blocks and digital block for an one-way SISO channel
In the reception RF unit, local oscillators $\mathrm{LO}_{\mathrm{TDD}}$ and $\mathrm{LO}_{\mathrm{FDD}}$ allow the RF to $\mathrm{IF}_{1}$ conversion by taking into account the TDD and FDD modes which use different frequency bandwidths. Then, the third local oscillator $\mathrm{LO}_{1}$ allows the $\mathrm{IF}_{1}$ to $\mathrm{IF}_{2}$ conversion. The $\mathrm{IF}_{2}$ pass-band filter must perform a good rejection of the out-of-band frequencies to avoid aliasing problems. The local oscillator $\mathrm{LO}_{2}$ and the Low Pass Filter give a low-pass signal. After the digital block, the transmission RF unit converts, with the equivalent steps, the low-pass version of the $\mathrm{y}_{\mathrm{j}}(\mathrm{t}, \tau)$ signal into a RF signal. The final RF amplifier restores the proper signal level by taking into account the attenuation introduced by AGC block.

\section{Digital Block}

By using the Nyquist-Shannon theorem and the expected performances of the RF/IF filters, the sampling frequency $f_{e}$ was chosen as $\mathrm{f}_{\mathrm{e}}>2 \mathrm{~B}$, i.e. $40 \mathrm{MHz}$ for UMTS systems and 50 $\mathrm{MHz}$ for WLAN systems. This choice allows a reasonable low sampling rate and avoids the aliasing problems.

According to the considered propagation environments, Table I summarizes some useful parameters.

TABLE I

SIMULATOR PARAMETERS

\begin{tabular}{cccrl}
\hline \hline & Environment & Cell Size & $\mathrm{N}\left(2^{\mathrm{n}}\right)$ & $\mathrm{W}_{\mathrm{t}}(\mu \mathrm{s})$ \\
\hline \multirow{2}{*}{ UMTS } & Rural & $2-20 \mathrm{~km}$ & 512 & 12,8 \\
& Urban & $0.4-2 \mathrm{~km}$ & 128 & 3,2 \\
& Indoor & $20-400 \mathrm{~m}$ & 32 & 0,8 \\
\hline & A office & $40 \mathrm{~m}$ & 32 & 0,64 \\
& B indoor & $50-150 \mathrm{~m}$ & 64 & 1,28 \\
WLAN, & C indoor & $50-150 \mathrm{~m}$ & 64 & 1,28 \\
Hiperlan2 & D indoor & $50-150 \mathrm{~m}$ & 64 & 1,28 \\
& E outdoor & $50-150 \mathrm{~m}$ & 128 & 2,56 \\
\hline \hline
\end{tabular}

For these various environments, the size of the FFT blocks is estimated by:

$$
\mathrm{N}=\frac{\mathrm{W}_{\mathrm{t}}}{\mathrm{T}_{\mathrm{e}}}=\mathrm{W}_{\mathrm{t}} \mathrm{f}_{\mathrm{e}}
$$

where $\mathrm{W}_{\mathrm{t}}$ represents the width of the effective time window of the impulse response of the propagation channel, i.e. the width of the time-interval where the impulse response can be considered not null. The final value $\mathrm{N}$ is the closest $2^{\mathrm{n}}$ value. The resulting $\mathrm{W}_{\mathrm{t}}$ is also given in Table $\mathrm{I}$.

For each environment, the maximum Doppler frequency can be calculated from the mobile speed and the carrier frequency. The coherence time can then be computed.

As shown in Fig. 9, the Fourier transform of the sampled low-pass input signal obtained at the output of the FFT block is multiplied by the channel frequency response $H_{i, j}(t, f)$, including the correction of errors introduced by the filters of the reception RF unit. Finally, from the resulting MIMO signal $Y_{j}(t, f)$ representing the sum of all SISO $Y_{i, j}(t, f)$ signals, the inverse Fourier transform (IFT block) gives the low-pass output signal $\mathrm{y}_{\mathrm{j}}(\mathrm{t}, \tau)$ witch represents the input of the transmission RF unit. 


\section{The Ptolemy Simulator}

During the SIMPAA project, a software propagation simulator for a SISO channel was produced with PTOLEMY development environment. PTOLEMY is an opened graphical design environment for heterogeneous systems. This software simulator makes it possible to define and design the various blocks of a radio communication system (filters, modulators, FFT blocks, etc).

Fig. 10 presents the PTOLEMY block diagram of the baseband simulator for one SISO channel. Two main parts can be identified: the lower part corresponding to the channel impulse response and the input signal part with the frequency domain multiplication.

The digital block of a one-way SISO channel calculates the Fourier transform of the input signal and computes, for each output SISO channel, the product between this Fourier transform and the corresponding frequency response resulting from the channel modeling.

At the output of each SISO channel, an inverse Fourier transform gives the complex envelope of its impulse response. The signal is then transposed to IF to supply the RF block. For the $\mathrm{j}^{\text {th }}$ receiving antenna, the signal $\mathrm{y}_{\mathrm{j}}(\mathrm{t}, \tau)$ provided by the simulator is, in fact, the sum of $\mathrm{N}_{\mathrm{T}}$ products corresponding to $\mathrm{N}_{\mathrm{T}}$ SISO channels.

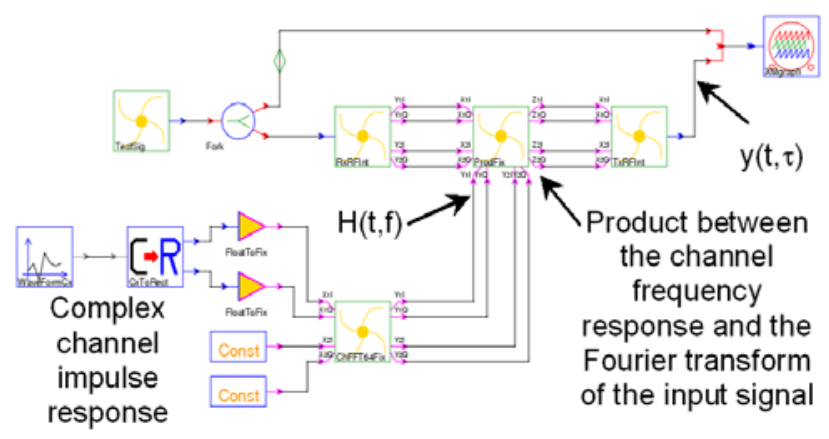

Fig. 10. PTOLEMY SISO channel simulation.

\section{A. Channel Impulse Responses}

This block must produce the complex envelope of the channel impulse response. The first stage consists in breaking up this complex impulse response in real and imaginary parts, followed by the Fourier transform. Thus, the baseband channel frequency response is obtained.

\section{B. Input Signal}

The input signal, provided by the TestSig block, is an IF sinusoidal testing signal. In the RxRFint block, this signal is divided into I (In-phase) and Q (Quadrature phase) components by its down-converter to baseband. Then, a FFT ensures the generation of the Fourier transform of the input signal. The product between the channel frequency response and the input signal Fourier transform represents the Fourier transform of the channel output signal. An inverse FFT makes it possible to obtain the complex envelope of channel response. Lastly, the signal is up-converted from baseband to IF to supply the RF block.

\section{CONCLUSION}

SIMPAA2 project has two main objectives. The first objective concerns the design and the realization of the hardware simulator by using a prototyping platform containing programmable circuits and/or very fast signal processors with a very large scale of integration. The second objective concerns the reliable MIMO channel models which must supply the digital block.

After a comparative study, in order to reduce the complexity of the digital block, the output signal of the MIMO simulator will be computed in the frequency domain. A software propagation simulator for a SISO channel was therefore produced by using the PTOLEMY development environment. This work will be continued to complete the design of the architecture of the hardware simulator by the choice of the number of bits per sample, data format (floatingpoint, fixed-point), minimization of the latency of the digital block, etc.

In order to obtain realistic models for different propagation environments, a MIMO channel sounder, realized by IETR was already used to perform several SISO and MIMO measurement campaigns. The analysis of the resulting data allowed a theoretical approach concerning the characterization of the MIMO propagation channel.

More measurement campaigns will be carried out with this MIMO channel sounder for various types of environments (indoor, outdoor, penetration), for both UMTS and WLAN frequency bands. The final objective of these measurements is to obtain realistic and reliable MIMO channel models in order to supply the digital block of the hardware simulator.

Lastly, the digital block of the MIMO hardware simulator will be implemented as an electronic module. A Graphical User Interface (GUI) will be also developed to allow the user to configure the channel parameters: environment type, channel model, time window, mobile speed, etc.

\section{REFERENCES}

[1] T. S. Rappaport, Wireless Communications Principles \& Practice, Prentice Hall, 1996.

[2] W.C. Jakes, Microwave Mobile Communications, John Wiley \& Sons, 1974.

[3] www.telecom.gouv.fr/rnrt/rnrt/projets/res d12 ap99.htm

[4] P.A. Bello, "Characterization of randomly time-variant linear channels", IEEE Trans. on Commun. Syst., vol. 11, December 1963, pp.360-393.

[5] J. Guillet, "Caractérisation et modélisation spatio-temporelles du canal de propagation radioélectrique dans le contexte MIMO." Thèse de doctorat en électronique, IETR, INSA de Rennes, juillet 2004. Available: http://tel.ccsd.cnrs.fr/documents/archives0/00/00/80/11.

[6] R. Cosquer, "Conception d'un sondeur de canal MIMO - Caractérisation du canal de propagation d'un point de vue directionnel et doublement directionnel. "Thèse de doctorat en électronique, IETR, INSA de Rennes, octobre 2004. Available: http:/tel.ccsd.cnrs.fr/documents/archives0/00/00/76/59. 